\title{
Motion of vortices outside a cylinder
}

\author{
Serdar Tulu and Oguz Yilmaz ${ }^{\text {a) }}$ \\ Department of Mathematics, Izmir Institute of Technology, Urla, Izmir 35430, Turkey
}

(Received 14 July 2010; accepted 16 September 2010; published online 2 November 2010)

\begin{abstract}
The problem of motion of the vortices around an oscillating cylinder in the presence of a uniform flow is considered. The Hamiltonian for vortex motion for the case with no uniform flow and stationary cylinder is constructed, reduced, and constant Hamiltonian (energy) curves are plotted when the system is shown to be integrable according to Liouville. By adding uniform flow to the system and by allowing the cylinder to vibrate, we model the natural vibration of the cylinder in the flow field, which has applications in ocean engineering involving tethers or pipelines in a flow field. We conclude that in the chaotic case forces on the cylinder may be considerably larger than those on the integrable case depending on the initial positions of vortices and that complex phenomena such as chaotic capture and escape occur when the initial positions lie in a certain region.
\end{abstract}

(C) 2010 American Institute of Physics. [doi:10.1063/1.3497915]

The investigation of point vortices in domains with boundaries is a popular research area with possible applications in engineering and physics such as the interaction of tension leg platforms or semisubmersibles with vortices in a uniform flow field. Integrable and chaotic cases of vortex motion are investigated in this paper. Chaotic motion of vortices is not only interesting in its own right but also it has some practical consequences such as causing increase on forces acting on the cylinder. Integrability in dynamical systems is very rare. Even if the system is integrable, it is not an easy task to reduce the system to lower dimensions. In this study, we find an integrable system of vortices and reduce it to two space dimensions and plot the constant Hamiltonian curves.

\section{INTRODUCTION}

We consider two different problems: the problem of fluid advection excited by two vortices and a uniform flow in the presence of a cylinder and the problem of motion of the vortices around the cylinder where a perturbation of the cylinder is also considered. Milne-Thomson's circle theorem ${ }^{1}$ is used to determine the flow field. In the fluid advection problem forces are calculated by the Blasius theorem. The effect of the independent circulation around the cylinder on force is investigated.

The Hamiltonian for vortex motion is constructed, reduced, and constant Hamiltonian (energy) curves are plotted when the system is shown to be integrable according to Liouville. By adding uniform flow to the system and by allowing the cylinder to vibrate, we model the natural vibration of the cylinder in the flow field, which has applications in ocean engineering involving tethers or pipelines in a flow field.

In this paper we consider several cases involving vortices, uniform flow, a cylinder, and an oscillatory perturbation

\footnotetext{
${ }^{\text {a) }}$ Author to whom correspondence should be addressed. Electronic mail: oguzyilmaz@iyte.edu.tr.
}

to the cylinder. In all cases first we deal with the fluid motion problem excited by the vortices and the uniform flow and then we consider the vortex motion problem. First we study the case of a stationary cylinder and two vortices and we see that the motion of vortices in this case is integrable when the vortices have equal strength. In the second case where a uniform flow is added to the system of two vortices and a cylinder, the symmetry is destroyed by the uniform flow and the motion of vortices is probably not integrable. The last case we consider involves the oscillatory perturbation of the cylinder, uniform flow, and vortices, and this case has applications in vortex induced oscillations of tethers. We demonstrate that chaos exist for certain parameter values and this results in higher forces exerted by the fluid on the cylinder.

In most vortex induced vibration problems, ${ }^{2-4}$ numerical and semianalytical techniques have been used to estimate stability, modes of oscillations, displacement, and vortex forces. The hydrodynamic interaction between vortices and an oscillating cylinder in the presence of a uniform flow will be considered and it may be used in understanding vortex induced vibration problems.

Hydrodynamic interaction problem has been studied by many authors in connection with free surface waves and surface piercing vertical cylinders (see, for example, Refs. 5 and 6). The basic idea behind these studies is that incoming waves can be decomposed into modes and diffracted waves from cylinders can be related to these modes and then the interaction takes place when we relate the coordinate systems at the center of each cylinder by using addition theorems. A similar idea was used by Pashaev and Yilmaz ${ }^{7}$ to find the interaction between cylinders and a vortex in the twodimensional plane. There are other methods such as Abelian function theory and conformal mapping techniques used in solving vortex body interaction problems (see Refs. 8-11).

To the best knowledge of authors, there are no results in the literature studying two vortices in the presence of a uniform flow and a vibrating cylinder. It is known that in unbounded plane, the motion of three vortices is integrable ${ }^{12}$ 
and the motion of four vortices is integrable for special arrangements of vortices. ${ }^{13-15}$ Vortices in annular regions have also been investigated by many authors. Johnson and McDonald $^{16}$ solved the problem in terms of elliptic functions. Analysis of the same problem by the method of images in terms of the q-calculus has been examined by Pashaev and Yilmaz. ${ }^{8}$ Advection problem of a point vortex in closed domains has been studied by Zannetti and Franzese. ${ }^{17}$ They use canonical transformations to eliminate the time dependence of the Hamiltonian.

\section{FLUID ADVECTION AND FORCES ON THE CYLINDER}

Since the problems we consider are on the twodimensional plane, we shall frequently make use of the theory of complex analytic functions. Also, MilneThomson's ${ }^{1}$ circle theorem will be very useful when we consider a single cylinder. We rewrite the circle theorem for the convenience of the reader.

Theorem 1: Milne-Thomson circle theorem. Let there be irrotational two-dimensional flow of incompressible inviscid fluid in the z-plane. Let there be no rigid boundaries and let the complex potential of the flow be $f(z)$, where the singularities of $f(z)$ are all at a distance greater than a from the origin. If a circular cylinder, typified by its cross-section the circle $C,|z|=a$, be introduced into the field of flow, the complex potential becomes

$$
\omega=f(z)+\overline{f\left(\frac{a^{2}}{\bar{z}}\right)}=f(z)+\bar{f}\left(\frac{a^{2}}{z}\right),
$$

with (i) the same singularities as $f(z)$ in $|z|>a$ and (ii) $|z|=a$ as a streamline.

The proof is easy once we notice that on the cylinder the complex potential becomes real so that the circle is a streamline.

We formulate the problem of two vortices, a uniform flow, and a stationary cylinders in unbounded twodimensional domain. In what follows $z$ denotes the complex variable $x+i y$. We assume that a cylinder of radius $a$ is placed at the origin in an unbounded two-dimensional fluid domain where two point vortices with strengths $\kappa_{1}, \kappa_{2}$ are located outside the cylinder at points $z_{01}, z_{02}$, respectively. There is a uniform flow with velocity $u_{0}$ in the negative $y$ direction at infinity. The complex potential is given by the circle theorem of Milne-Thomson, ${ }^{1}$

$$
\begin{aligned}
\omega= & i u_{0} z+i \kappa_{1} \log \left(z-z_{01}\right)+i \kappa_{2} \log \left(z-z_{02}\right)-i u_{0} \frac{a^{2}}{z} \\
& -i \kappa_{1} \log \left(\frac{a^{2}}{z}-\bar{z}_{01}\right)-i \kappa_{2} \log \left(\frac{a^{2}}{z}-\bar{z}_{02}\right) .
\end{aligned}
$$

The first three terms in Eq. (1) are complex potentials of the two vortices and the uniform flow and the last three terms represent the effect of the cylinder. It is easy to see that on the cylinder, the imaginary part of the complex potential, the stream function, vanishes hence the boundary condition is satisfied. After some simple manipulations we can rewrite the complex potential as

$$
\begin{aligned}
\omega= & i u_{0}\left(z-\frac{a^{2}}{z}\right)+i \kappa_{1} \log \left(z-z_{01}\right)-i \kappa_{1} \log \left(z-z_{1}^{\prime}\right) \\
& +i \kappa_{1} \log (z)-i \kappa_{1} \log \left(-\bar{z}_{01}\right)+i \kappa_{2} \log \left(z-z_{02}\right) \\
& -i \kappa_{2} \log \left(z-z_{2}^{\prime}\right)+i \kappa_{2} \log (z)-i \kappa_{2} \log \left(-\bar{z}_{02}\right)
\end{aligned}
$$

where $z_{1}^{\prime}=a^{2} / \bar{z}_{01}$ and $z_{2}^{\prime}=a^{2} / \bar{z}_{02}$. We notice that in Eq. (2) there are two images for each vortex: one at the inverse point of the vortex with respect to the cylinder, $a^{2} / \bar{z}_{01}$, another one at the origin. While the first image has the negative strength, the latter has the positive strength.

We should remark that the image vortex at the origin is not necessary to satisfy the boundary condition, for a vortex at the center of the cylinder will give only tangential component on the boundary of the cylinder. The effect of the image at the center which has the same strength but opposite sign to the one at the inverse point is to produce zero circulation about the cylinder. It is possible to generalize the problem by making the strength of the image at the center different from the one at the inverse point and therefore to have nonzero circulation about the cylinder. However, as we shall see, zero circulation does not necessarily mean zero force.

The complex velocity is obtained by differentiating Eq. (2),

$$
\begin{aligned}
\bar{V}= & i u_{0}\left(1+\frac{a^{2}}{z^{2}}\right)+\frac{i \kappa_{1}}{z-z_{01}}-\frac{i \kappa_{1}}{z-z_{1}^{\prime}}+\frac{i \kappa_{1}}{z}+\frac{i \kappa_{2}}{z-z_{02}} \\
& -\frac{i \kappa_{2}}{z-z_{2}^{\prime}}+\frac{i \kappa_{2}}{z}
\end{aligned}
$$

where $\bar{V}=d \omega / d z=u-i v$, with $u$ and $v$ being rectangular components of velocity. Henceforth, we shall work with complex velocity and avoid dealing with the multivalued function log. In Fig. 1 the flow field is shown when the two vortices have the same strength and $u_{0}=0$. We see that at the bisector of the line joining the centers of two vortices, there is a stagnation point. Whereas in Fig. 2 where total strength of vortices vanishes and $u_{0}=0$ there are no stagnation points. Also the force acting on the cylinder is 80 times higher in the latter case.

Before we calculate the force on the cylinder, we shall generalize Eq. (3) by making the strength at the center of the cylinder arbitrary, 


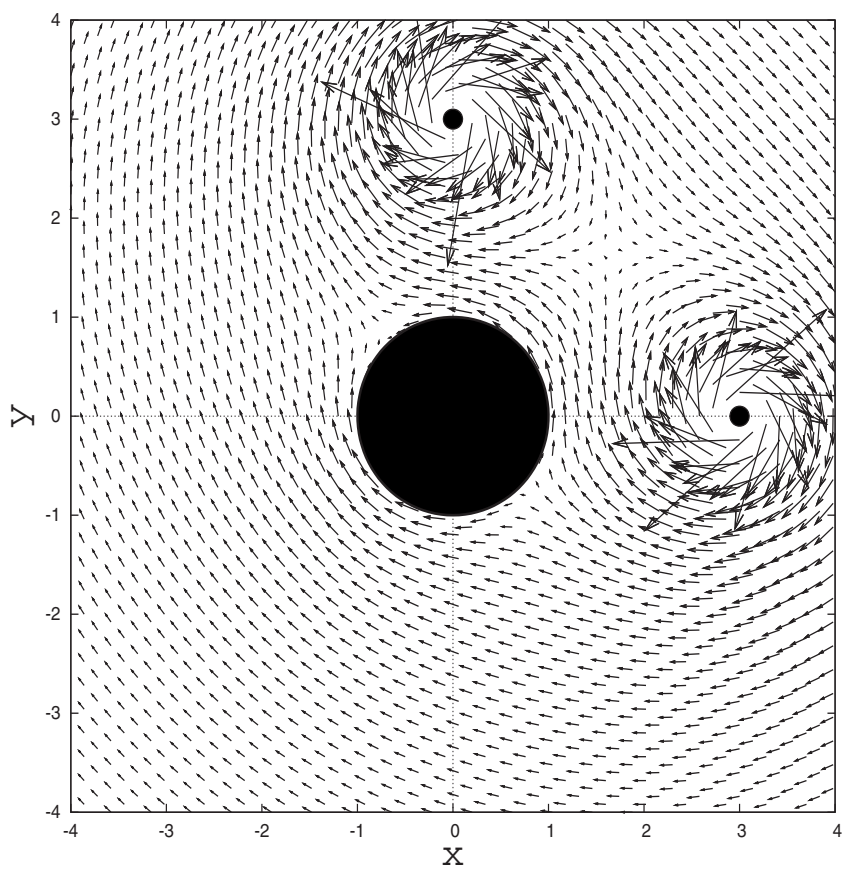

FIG. 1. Velocity distribution about a cylinder at the origin with two vortices of unit strength. Velocity vectors are scaled down by the factor of 1.3.

$$
\begin{aligned}
\bar{V}= & i u_{0}\left(1+\frac{a^{2}}{z^{2}}\right)+\frac{i \kappa_{1}}{z-z_{01}}-\frac{i \kappa_{1}}{z-z_{1}^{\prime}}+\frac{i \kappa_{0}}{z}+\frac{i \kappa_{2}}{z-z_{02}} \\
& -\frac{i \kappa_{2}}{z-z_{2}^{\prime}} .
\end{aligned}
$$

By Blasius' theorem, the force on the system is given by

$$
\begin{aligned}
\bar{F}= & \frac{i \rho}{2} \int_{|z|=a} \bar{V}^{2} d z=2 \pi \rho \kappa_{1}^{2}\left(\frac{1}{z_{01}-z_{1}^{\prime}}\right)+2 \pi \rho \kappa_{2}^{2}\left(\frac{1}{z_{02}-z_{2}^{\prime}}\right) \\
& +2 \pi \rho \kappa_{1} \kappa_{2}\left(\frac{1}{z_{01}-z_{2}^{\prime}}+\frac{1}{z_{02}-z_{1}^{\prime}}\right)-2 \pi \rho \kappa_{0}\left(\frac{\kappa_{1}}{z_{01}}+\frac{\kappa_{2}}{z_{02}}\right) \\
& -2 \pi \rho u_{0}\left(\kappa_{1}\left(\frac{a^{2}}{z_{01}^{2}}+1\right)+\kappa_{2}\left(\frac{a^{2}}{z_{02}^{2}}+1\right)-\kappa_{0}\right) .
\end{aligned}
$$

Notice that the circulation around the cylinder depends on the value of $\kappa_{0}$,

$$
\Gamma=2 \pi\left(\kappa_{1}-\kappa_{0}+\kappa_{2}\right)
$$

when $\kappa_{0}=\kappa_{1}+\kappa_{2}$, circulation would be zero, as expected.

We consider two cases: (1) $u_{0}=0$ and (2) $u_{0} \neq 0$.

(1) When there is no uniform flow, a simple analysis of Eq. (5) shows that force on the cylinder becomes zero under certain conditions; when vortices have the same strength $\left(\kappa_{1}=\kappa_{2}\right)$, are equidistant from the center of the cylinder, and are placed symmetrically with respect to the center. Maximum force would occur in the limiting case when the same-sign vortices collide. Instead of $\kappa_{1}=\kappa_{2}$, if we assume that $\kappa_{1}=-\kappa_{2}$, then $\kappa_{0}$ must be zero for force to be zero under the same conditions as before.

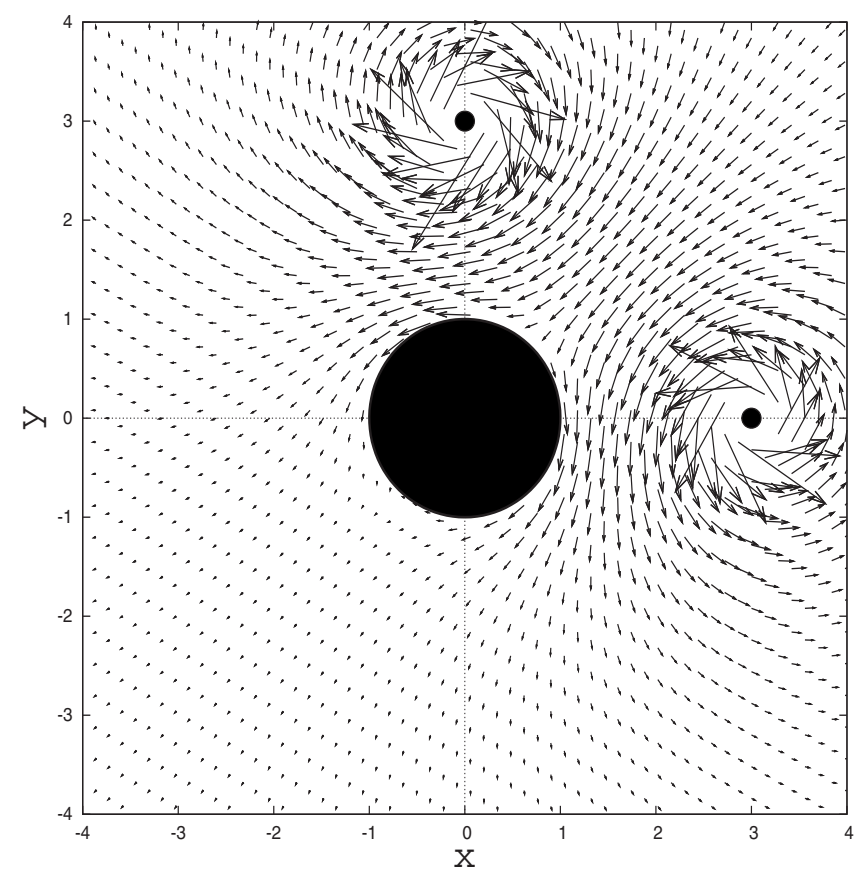

FIG. 2. Velocity distribution about a cylinder at the origin with two vortices of vanishing total strength. Velocity vectors are scaled down by the factor of 1.3 .

(2) However, when there is a uniform flow and $\kappa_{1}=\kappa_{2}$, force is never zero. If $\kappa_{1}=-\kappa_{2}$ then for force to be zero vortices must be placed symmetrically with respect to the center of the cylinder and $\kappa_{0}=0$.

\section{MOTION OF VORTICES}

We consider the cases with and without uniform flow separately for two cases have completely different characteristics. Without uniform flow, there is symmetry in the system and the vortex motion is integrable. However, with uniform flow the symmetry is lost and the behavior is chaotic.

\section{A. The case of two vortices and a cylinder}

Consider two vortices of strengths $\kappa_{1}, \kappa_{2}$ at $z_{01}, z_{02}$ and a cylinder of radius $a$ at the origin. The complex velocity is given by

$$
\bar{V}=\frac{i \kappa_{1}}{z-z_{01}}-\frac{i \kappa_{1}}{z-\frac{a^{2}}{\bar{z}_{01}}}+\frac{i \kappa_{2}}{z-z_{02}}-\frac{i \kappa_{2}}{z-\frac{a^{2}}{\bar{z}_{02}}}+\frac{i \kappa_{0}}{z} .
$$

The motion of vortex at $z_{01}$ can be found by replacing $z$ by $z_{01}$ in Eq. (6), and omitting the effect of the vortex itself,

$$
\frac{d \bar{z}_{01}}{d t}=\frac{i \kappa_{2}}{z_{01}-z_{02}}-\frac{i \kappa_{1} \bar{z}_{01}}{\left|z_{01}\right|^{2}-a^{2}}-\frac{i \kappa_{2} \bar{z}_{02}}{z_{01} \bar{z}_{02}-a^{2}}+\frac{i \kappa_{0}}{z_{01}} .
$$

The motion of vortex at $z_{02}$ can be found similarly

$$
\frac{d \bar{z}_{02}}{d t}=\frac{i \kappa_{1}}{z_{02}-z_{01}}-\frac{i \kappa_{2} \bar{z}_{02}}{\left|z_{02}\right|^{2}-a^{2}}-\frac{i \kappa_{1} \bar{z}_{01}}{z_{02} \bar{z}_{01}-a^{2}}+\frac{i \kappa_{0}}{z_{02}} .
$$

Letting $z_{01}=x_{01}+i y_{01}$ and $z_{02}=x_{02}+i y_{02}$, we have a system of four coupled ordinary nonlinear differential equations, 


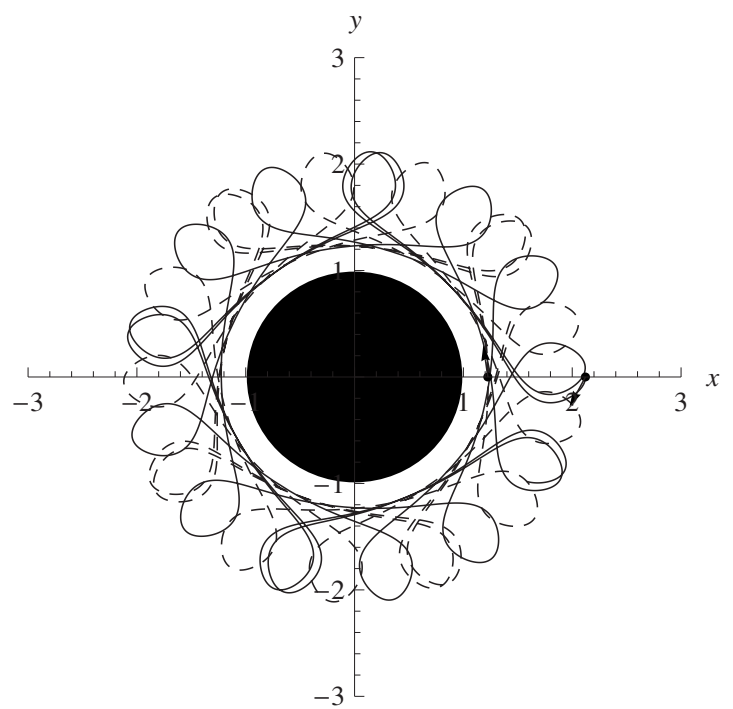

FIG. 3. Motion of vortices of unit strength around the cylinder at the origin with initial vortex starting positions being $(1.322876,0),(2.061553,0)$ and $\kappa_{0}=\kappa_{1}+\kappa_{2}$. Continuous and dashed lines denote the trajectories of vortices. The initial points correspond to the point B in Fig. 5.

$$
\begin{aligned}
\frac{d x_{01}}{d t}= & \frac{\kappa_{2}\left(y_{01}-y_{02}\right)}{\left|z_{01}-z_{02}\right|^{2}}-\frac{\kappa_{1} y_{01}}{\left|z_{01}\right|^{2}-a^{2}} \\
& +\frac{\kappa_{2}\left(a^{2} y_{02}-y_{01}\left|z_{02}\right|^{2}\right)}{\left|z_{01} \bar{z}_{02}-a^{2}\right|^{2}}+\frac{\kappa_{0} y_{01}}{\left|z_{01}\right|^{2}},
\end{aligned}
$$

$$
\begin{aligned}
\frac{d y_{01}}{d t}= & -\frac{\kappa_{2}\left(x_{01}-x_{02}\right)}{\left|z_{01}-z_{02}\right|^{2}}+\frac{\kappa_{1} x_{01}}{\left|z_{01}\right|^{2}-a^{2}} \\
& -\frac{\kappa_{2}\left(a^{2} x_{02}-x_{01}\left|z_{02}\right|^{2}\right)}{\left|z_{01} \bar{z}_{02}-a^{2}\right|^{2}}-\frac{\kappa_{0} x_{01}}{\left|z_{01}\right|^{2}},
\end{aligned}
$$

$$
\begin{aligned}
\frac{d x_{02}}{d t}= & \frac{\kappa_{1}\left(y_{02}-y_{01}\right)}{\left|z_{02}-z_{01}\right|^{2}}-\frac{\kappa_{2} y_{02}}{\left|z_{02}\right|^{2}-a^{2}} \\
& +\frac{\kappa_{1}\left(a^{2} y_{01}-y_{02}\left|z_{01}\right|^{2}\right)}{\left|z_{02} \bar{z}_{01}-a^{2}\right|^{2}}+\frac{\kappa_{0} y_{02}}{\left|z_{02}\right|^{2}},
\end{aligned}
$$

$$
\begin{aligned}
\frac{d y_{02}}{d t}= & -\frac{\kappa_{1}\left(x_{02}-x_{01}\right)}{\left|z_{02}-z_{01}\right|^{2}}+\frac{\kappa_{2} x_{02}}{\left|z_{02}\right|^{2}-a^{2}} \\
& -\frac{\kappa_{1}\left(a^{2} x_{01}-x_{02}\left|z_{01}\right|^{2}\right)}{\left|z_{02} \bar{z}_{01}-a^{2}\right|^{2}}-\frac{\kappa_{0} x_{02}}{\left|z_{02}\right|^{2}} .
\end{aligned}
$$

The Hamiltonian of vortex motion easily can be written down by inspection,

$$
\begin{aligned}
H= & \frac{\kappa_{1} \kappa_{2}}{2} \log \left(\left(x_{01}-x_{02}\right)^{2}+\left(y_{01}-y_{02}\right)^{2}\right)-\frac{\kappa_{1}^{2}}{2} \log \left(x_{01}^{2}+y_{01}^{2}\right. \\
& \left.-a^{2}\right)-\frac{\kappa_{2}^{2}}{2} \log \left(x_{02}^{2}+y_{02}^{2}-a^{2}\right)+\frac{\kappa_{1} \kappa_{0}}{2} \log \left(x_{01}^{2}+y_{01}^{2}\right) \\
& +\frac{\kappa_{2} \kappa_{0}}{2} \log \left(x_{02}^{2}+y_{02}^{2}\right)-\frac{\kappa_{1} \kappa_{2}}{2} \log \left(\left(y_{01} x_{02}-x_{01} y_{02}\right)^{2}\right. \\
& \left.+\left(y_{01} y_{02}+x_{01} x_{02}-a^{2}\right)^{2}\right) .
\end{aligned}
$$
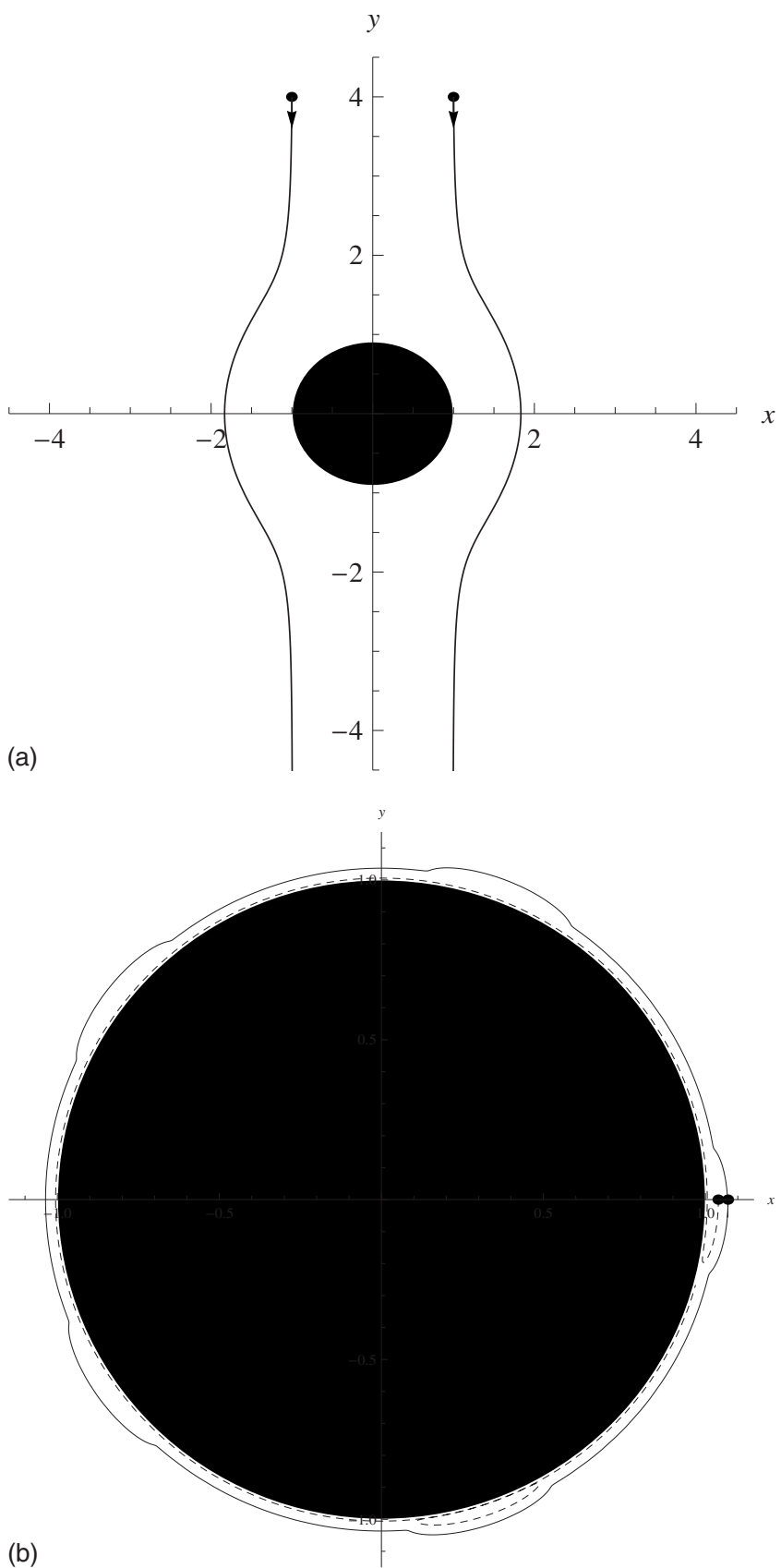

FIG. 4. (a) Motion of vortices with vanishing total strength around the cylinder at the origin with $\kappa_{0}=\kappa_{1}+\kappa_{2}$. Initial vortex positions are $(1,4)$ and $(-1,4)$. (b) Motion of vortices with vanishing total strength around the cylinder at the origin with $\kappa_{0}=\kappa_{1}+\kappa_{2}$. Initial positions are $(1.07,0)$ and $(1.04,0)$. Continuous and dashed lines denote the trajectories of vortices. Small solid dots indicate the initial positions of vortices.

Then it is easy to check that the motion equations can be derived using the Hamiltonian,

$$
\frac{d x_{0 i}}{d t}=\frac{1}{\kappa_{i}} \frac{\partial H}{\partial y_{0 i}}, \quad \frac{d y_{0 i}}{d t}=-\frac{1}{\kappa_{i}} \frac{\partial H}{\partial x_{0 i}}, \quad i=1,2 .
$$

We know that the Hamiltonian is an integral constant. We shall show that the angular momentum,

$$
I=\sum_{k=1}^{2} \kappa_{k} z_{0 k} \bar{z}_{0 k},
$$

is also an integral constant. Since 


$$
\begin{aligned}
\frac{d I}{d t}= & \frac{d}{d t}\left(\sum_{k=1}^{2} \kappa_{k} z_{0 k} \bar{z}_{0 k}\right)=\kappa_{1}\left(2 x_{01} \frac{d x_{01}}{d t}+2 y_{01} \frac{d y_{01}}{d t}\right) \\
& +\kappa_{2}\left(2 x_{02} \frac{d x_{02}}{d t}+2 y_{02} \frac{d y_{02}}{d t}\right)=0
\end{aligned}
$$

angular momentum is independent of time. The Poisson bracket of the Hamiltonian and the angular momentum can be calculated as follows:

$$
\begin{aligned}
\{I, H\}= & \frac{\partial I}{\partial x_{01}} \frac{\partial H}{\partial y_{01}}-\frac{\partial I}{\partial y_{01}} \frac{\partial H}{\partial x_{01}}+\frac{\partial I}{\partial x_{02}} \frac{\partial H}{\partial y_{02}}-\frac{\partial I}{\partial y_{02}} \frac{\partial H}{\partial x_{02}} \\
= & 2 \kappa_{1} \kappa_{2}\left(\kappa_{1}-\kappa_{2}\right) \frac{x_{02} y_{01}}{\left|z_{01}-z_{02}\right|^{2}}+2 \kappa_{1} \kappa_{2}\left(\kappa_{2}-\kappa_{1}\right) \\
& \times \frac{x_{01} y_{02}}{\left|z_{01}-z_{02}\right|^{2}}+2 \kappa_{1} \kappa_{2}\left(\kappa_{1}-\kappa_{2}\right) \frac{a^{2} x_{01} y_{02}}{\left|z_{01} \bar{z}_{02}-a^{2}\right|^{2}} \\
& +2 \kappa_{1} \kappa_{2}\left(\kappa_{2}-\kappa_{1}\right) \frac{a^{2} x_{02} y_{01}}{\left|z_{01} \bar{z}_{02}-a^{2}\right|^{2}} .
\end{aligned}
$$

When $\kappa_{1}=\kappa_{2}$ we obtain that $\{I, H\}=0$ then the Hamiltonian and the angular momentum are in involution. Hence the system is integrable according to Liouville.

Next we consider two numerical examples where $\kappa_{1}= \pm \kappa_{2}=1$ and $a=1$, then the motion of vortices are depicted in the Figs. 3 and 4. We see that when the vortices have the same strength, they rotate around each other and also around the cylinder (Fig. 3), whereas in the latter case vortices either translate with a uniform velocity [Fig. 4(a)] or rotate around the cylinder [Fig. 4(b)].

Since the system is integrable we shall try to reduce the system from four space variables to two space variables and then plot the constant Hamiltonian curves which gives information about the dynamical system in question. In order to put the motion equations in the usual Hamiltonian form, we let $p_{i}=\sqrt{\left|\kappa_{i}\right|} x_{0 i} s_{i}$ and $q_{i}=\sqrt{\left|\kappa_{i}\right|} y_{0 i}$, where $s_{i}=\operatorname{sign}\left(\kappa_{i}\right)$, then we have

$$
\frac{d p_{i}}{d t}=\frac{\partial H^{\prime}}{\partial q_{i}}, \quad \frac{d q_{i}}{d t}=-\frac{\partial H^{\prime}}{\partial p_{i}}, \quad i=1,2,
$$

where

$$
\begin{aligned}
H^{\prime}= & \frac{\kappa_{1} \kappa_{2}}{2} \log \left(\frac{p_{1}^{2}+q_{1}^{2}}{\left|\kappa_{1}\right|}+\frac{p_{2}^{2}+q_{2}^{2}}{\left|\kappa_{2}\right|}-\frac{2 p_{1} p_{2}}{\sqrt{\left|\kappa_{1} \kappa_{2}\right|} s_{1} s_{2}}-\frac{2 q_{1} q_{2}}{\sqrt{\left|\kappa_{1} \kappa_{2}\right|}}\right)-\frac{\kappa_{1}^{2}}{2} \log \left(\frac{p_{1}^{2}+q_{1}^{2}}{\left|\kappa_{1}\right|}-a^{2}\right)-\frac{\kappa_{2}^{2}}{2} \log \left(\frac{p_{2}^{2}+q_{2}^{2}}{\left|\kappa_{2}\right|}-a^{2}\right) \\
& +\frac{\kappa_{1} \kappa_{0}}{2} \log \left(\frac{p_{1}^{2}+q_{1}^{2}}{\left|\kappa_{1}\right|}\right)+\frac{\kappa_{2} \kappa_{0}}{2} \log \left(\frac{p_{2}^{2}+q_{2}^{2}}{\left|\kappa_{2}\right|}\right)-\frac{\kappa_{1} \kappa_{2}}{2} \log \left(\frac{q_{1}^{2} p_{2}^{2}+p_{1}^{2} q_{2}^{2}+q_{1}^{2} q_{2}^{2}+p_{1}^{2} p_{2}^{2}}{\left|\kappa_{1} \kappa_{2}\right|}-2 a^{2}\left(\frac{q_{1} q_{2}}{\sqrt{\left|\kappa_{1} \kappa_{2}\right|}}+\frac{p_{1} p_{2}}{\sqrt{\left|\kappa_{1} \kappa_{2}\right|} s_{1} s_{2}}\right)+a^{4}\right) .
\end{aligned}
$$

Then we employ polar coordinates $p_{i}^{2}+q_{i}^{2}=2 R_{i}$ and $q_{i} / p_{i}=\tan P_{i}, i=1,2$, so the Hamiltonian and the motion equations become

$$
\begin{aligned}
H^{\prime \prime}= & \frac{\kappa_{1} \kappa_{2}}{2} \log \left(\frac{2 R_{1}}{\left|\kappa_{1}\right|}+\frac{2 R_{2}}{\left|\kappa_{2}\right|}-\frac{4 \sqrt{R_{1} R_{2}}}{\sqrt{\left|\kappa_{1} \kappa_{2}\right|}} s_{1} s_{2} \cos \left(P_{1}-s_{1} s_{2} P_{2}\right)\right)-\frac{\kappa_{1}^{2}}{2} \log \left(\frac{2 R_{1}}{\left|\kappa_{1}\right|}-a^{2}\right)-\frac{\kappa_{2}^{2}}{2} \log \left(\frac{2 R_{2}}{\left|\kappa_{2}\right|}-a^{2}\right)+\frac{\kappa_{1} \kappa_{0}}{2} \log \left(\frac{2 R_{1}}{\left|\kappa_{1}\right|}\right) \\
& +\frac{\kappa_{2} \kappa_{0}}{2} \log \left(\frac{2 R_{2}}{\left|\kappa_{2}\right|}\right)-\frac{\kappa_{1} \kappa_{2}}{2} \log \left(\frac{4 R_{1} R_{2}}{\left|\kappa_{1} \kappa_{2}\right|}-\frac{4 a^{2} \sqrt{R_{1} R_{2}}}{\sqrt{\left|\kappa_{1} \kappa_{2}\right|}} s_{1} s_{2} \cos \left(P_{1}-s_{1} s_{2} P_{2}\right)+a^{4}\right)
\end{aligned}
$$

and

$$
\frac{d R_{i}}{d t}=\frac{\partial H^{\prime \prime}}{\partial P_{i}}, \quad \frac{d P_{i}}{d t}=-\frac{\partial H^{\prime \prime}}{\partial R_{i}}, \quad i=1,2 .
$$

Finally, with the new set of canonical variables,

$$
\begin{aligned}
& Q_{1}\left(P_{1}, P_{2}\right)=P_{1}-s_{1} s_{2} P_{2}, \quad Q_{2}\left(P_{1}, P_{2}\right)=s_{2} P_{2}, \\
& G_{1}\left(R_{1}, R_{2}\right)=R_{1}, \quad G_{2}\left(R_{1}, R_{2}\right)=s_{1} R_{1}+s_{2} R_{2},
\end{aligned}
$$

the Hamiltonian becomes

$$
\begin{aligned}
K= & \frac{\kappa_{1} \kappa_{2}}{2} \log \left(\frac{2 G_{1}}{\left|\kappa_{1}\right|}+\frac{2 s_{2}\left(G_{2}-s_{1} G_{1}\right)}{\left|\kappa_{2}\right|}-\frac{4 s_{1} s_{2}}{\sqrt{\left|\kappa_{1} \kappa_{2}\right|}} \sqrt{G_{1} s_{2}\left(G_{2}-s_{1} G_{1}\right)} \cos Q_{1}\right)-\frac{\kappa_{1}^{2}}{2} \log \left(\frac{2 G_{1}}{\left|\kappa_{1}\right|}-a^{2}\right) \\
& -\frac{\kappa_{2}^{2}}{2} \log \left(\frac{2 s_{2}\left(G_{2}-s_{1} G_{1}\right)}{\left|\kappa_{2}\right|}-a^{2}\right)+\frac{\kappa_{1} \kappa_{0}}{2} \log \left(\frac{2 G_{1}}{\left|\kappa_{1}\right|}\right)+\frac{\kappa_{2} \kappa_{0}}{2} \log \left(\frac{2 s_{2}\left(G_{2}-s_{1} G_{1}\right)}{\left|\kappa_{2}\right|}\right) \\
& -\frac{\kappa_{1} \kappa_{2}}{2} \log \left(\frac{4 G_{1} s_{2}\left(G_{2}-s_{1} G_{1}\right)}{\left|\kappa_{1} \kappa_{2}\right|}-\frac{4 a^{2} s_{1} s_{2}}{\sqrt{\left|\kappa_{1} \kappa_{2}\right|}} \sqrt{G_{1} s_{2}\left(G_{2}-s_{1} G_{1}\right)} \cos Q_{1}+a^{4}\right),
\end{aligned}
$$




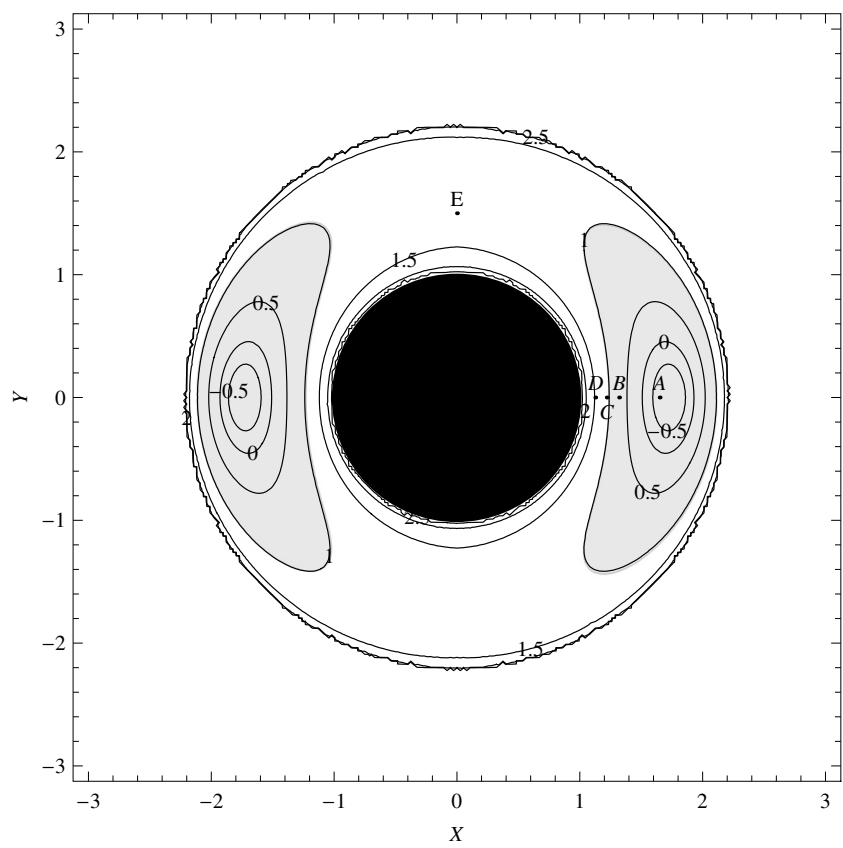

FIG. 5. The level curves for the Hamiltonian in $X Y$ coordinates. Vortices have unit strength and there is no net circulation about the cylinder; $\kappa_{0}=\kappa_{1}+\kappa_{2}$. The capture zone is shown as gray shaded region in the figure. The coordinates of the points are: $A=(1.65,0), B=(1.322876,0)$, $C=(1.2206,0), D=(1.15,0), E=(0,1.5)$.

and the corresponding motion equations are

$$
\begin{aligned}
& \frac{\partial K}{\partial Q_{1}}=\frac{d G_{1}}{d t}, \quad \frac{\partial K}{\partial G_{1}}=-\frac{d Q_{1}}{d t}, \\
& \frac{\partial K}{\partial G_{2}}=-\frac{d Q_{2}}{d t},
\end{aligned}
$$

and the angular momentum becomes

$$
\begin{aligned}
I= & \kappa_{1}\left(x_{01}^{2}+y_{01}^{2}\right)+\kappa_{2}\left(x_{02}^{2}+y_{02}^{2}\right)=\kappa_{1}\left(\frac{p_{1}^{2}+q_{1}^{2}}{\left|\kappa_{1}\right|}\right) \\
& +\kappa_{2}\left(\frac{p_{2}^{2}+q_{2}^{2}}{\left|\kappa_{2}\right|}\right)=2\left(s_{1} R_{1}+s_{2} R_{2}\right)=2 G_{2} .
\end{aligned}
$$

Since angular momentum is constant, the Hamiltonian is cyclic in $Q_{2}$ and its conjugate $G_{2}$, which is angular momentum, is constant. By employing these sets of canonical transformations, we reduce the system from four degrees of freedom to two degrees of freedom, $G_{1}$ and $Q_{1}$. The ranges for the variables $G_{1}, Q_{1}$ and the constant $G_{2}$ are $G_{1}>\left(a^{2} / 2\right)\left|\kappa_{1}\right|, \quad G_{2}>\left(a^{2} / 2\right)\left(s_{1}\left|\kappa_{1}\right|+s_{2}\left|\kappa_{2}\right|\right)$, and $-2 \pi<Q_{1}<2 \pi$.

We consider a numerical example when $\kappa_{1}=\kappa_{2}=1$ and $a=1$. In this case the level curves of the Hamiltonian are shown in Fig. 5 for $G_{2}=3$ in a coordinate system $(X, Y)$, where $X=\sqrt{2 R_{1}} \cos \left(P_{1}-P_{2}\right)$ and $Y=\sqrt{2 R_{1}} \sin \left(P_{1}-P_{2}\right)$. That implies we are measuring the distance of the first vortex to the origin in the coordinate system $(X, Y)$ that rotates with the second vortex. In fact, Fig. 5 corresponds to the Poincare map in the system $(p, q)$, where the angular momentum and the Hamiltonian are first integrals of motion. So the trajectories are plotted when the angular momentum and $P_{2}$ are

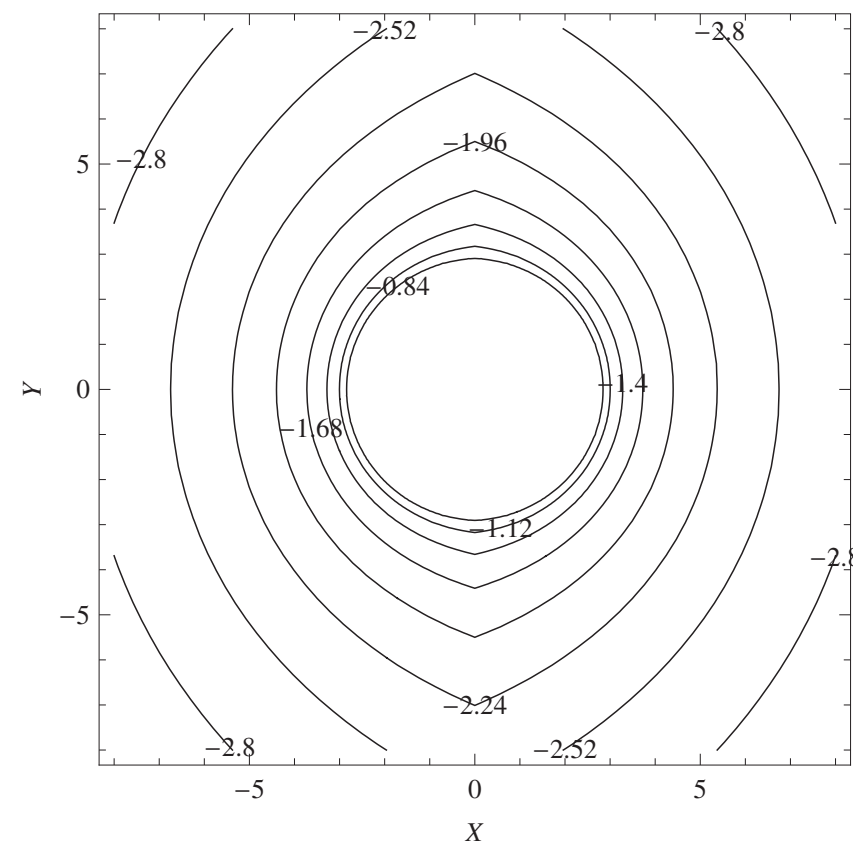

FIG. 6. The level curves for the Hamiltonian in XY coordinates. Vortices have opposite unit strengths and there is no net circulation around the cylinder; $\kappa_{0}=\kappa_{1}+\kappa_{2}$.

fixed. We also notice that, in Fig. 5, there are two fixed elliptic points at the points $(-\sqrt{3}, 0)$ and $(\sqrt{3}, 0)$ and that the trajectories are confined to the circles with radii of 1 and $\sqrt{5}$. The inner circle in Fig. 5 corresponds to the cylinder and the outer one exists since the total angular momentum is constant.

In Fig. 6, the level curves of the Hamiltonian are shown for the case when vortices have opposite strengths and $G_{2}=3$. We see that when the initial positions of vortices are close to the cylinder, they rotate around the cylinder [see Fig. 4(b)]. But when they start off far enough from the cylinder, they translate with uniform velocity [Fig. 4(a)]. Although we cannot prove the integrability directly in this case, it is obvious from Fig. 6 that the system is integrable.

\section{B. Motion of two vortices around a cylinder in the presence of a uniform flow}

Consider two vortices of strengths $\kappa_{1}, \kappa_{2}$ at $z_{01}, z_{02}$, a uniform flow with velocity along negative $y$ axis $u_{0}$ and a cylinder of radius $a$ at the origin. The complex potential is

$$
\begin{aligned}
\bar{V}= & i u_{0}\left(1+\frac{a^{2}}{z^{2}}\right)+\frac{i \kappa_{1}}{z-z_{01}}-\frac{i \kappa_{1}}{z-\frac{a^{2}}{\bar{z}_{01}}}+\frac{i \kappa_{2}}{z-z_{02}}-\frac{i \kappa_{2}}{z-\frac{a^{2}}{\bar{z}_{02}}} \\
& +\frac{i \kappa_{0}}{z} .
\end{aligned}
$$

The motion of vortices at $z_{01}$ and $z_{02}$ can be found easily, 


$$
\begin{aligned}
\frac{d \bar{z}_{01}}{d t}= & i u_{0}\left(1+\frac{a^{2}}{z_{01}}\right)+\frac{i \kappa_{2}}{z_{01}-z_{02}}-\frac{i \kappa_{1} \bar{z}_{01}}{\left|z_{01}\right|^{2}-a^{2}}-\frac{i \kappa_{2} \bar{z}_{02}}{z_{01} \bar{z}_{02}-a^{2}} \\
& +\frac{i \kappa_{0}}{\bar{z}_{01}}, \\
\frac{d \bar{z}_{02}}{d t}= & i u_{0}\left(1+\frac{a^{2}}{z_{02}}\right)+\frac{i \kappa_{1}}{z_{02}-z_{01}}-\frac{i \kappa_{2} \bar{z}_{02}}{\left|z_{02}\right|^{2}-a^{2}}-\frac{i \kappa_{1} \bar{z}_{01}}{z_{02} \bar{z}_{01}-a^{2}} \\
& +\frac{i \kappa_{0}}{\bar{z}_{02}} .
\end{aligned}
$$

Letting $z_{01}=x_{01}+i y_{01}$ and $z_{02}=x_{02}+i y_{02}$, we have a system of ordinary nonlinear differential equations,

$$
\begin{aligned}
\frac{d x_{01}}{d t}= & \frac{2 u_{0} a^{2} x_{01} y_{01}}{\left|z_{01}\right|^{4}}+\frac{\kappa_{2}\left(y_{01}-y_{02}\right)}{\left|z_{01}-z_{02}\right|^{2}}-\frac{\kappa_{1} y_{01}}{\left|z_{01}\right|^{2}-a^{2}}+\frac{\kappa_{0} y_{01}}{\left|z_{01}\right|^{2}} \\
& +\frac{\kappa_{2}\left(a^{2} y_{02}-y_{01}\left|z_{02}\right|^{2}\right)}{\left|z_{01} \bar{z}_{02}-a^{2}\right|^{2}}, \\
\frac{d y_{01}}{d t}= & u_{0}\left(\frac{a^{2} y_{01}^{2}-a^{2} x_{01}^{2}}{\left|z_{01}\right|^{4}}-1\right)-\frac{\kappa_{2}\left(x_{01}-x_{02}\right)}{\left|z_{01}-z_{02}\right|^{2}}-\frac{\kappa_{0} x_{01}}{\left|z_{01}\right|^{2}} \\
& +\frac{\kappa_{1} x_{01}}{\left|z_{01}\right|^{2}-a^{2}}-\frac{\kappa_{2}\left(a^{2} x_{02}-x_{01}\left|z_{02}\right|^{2}\right)}{\left|z_{01} \bar{z}_{02}-a^{2}\right|^{2}}, \\
\frac{d x_{02}}{d t}= & \frac{2 u_{0} a^{2} x_{02} y_{02}}{\left|z_{02}\right|^{4}}+\frac{\kappa_{1}\left(y_{02}-y_{01}\right)}{\left|z_{02}-z_{01}\right|^{2}}-\frac{\kappa_{2} y_{02}}{\left|z_{02}\right|^{2}-a^{2}}+\frac{\kappa_{0} y_{02}}{\left|z_{02}\right|^{2}} \\
& +\frac{\kappa_{1}\left(a^{2} y_{01}-y_{02}\left|z_{01}\right|^{2}\right)}{\left|z_{02} \bar{z}_{01}-a^{2}\right|^{2}}, \\
\frac{d y_{02}}{d t}= & u_{0}\left(\frac{a^{2} y_{02}^{2}-a^{2} x_{02}^{2}}{\left|z_{02}\right|^{4}}-1\right)-\frac{\kappa_{1}\left(x_{02}-x_{01}\right)}{\left|z_{02}-z_{01}\right|^{2}}-\frac{\kappa_{0} x_{02}}{\left|z_{02}\right|^{2}} \\
& +\frac{\kappa_{2} x_{02}}{\left|z_{02}\right|^{2}-a^{2}}-\frac{\kappa_{1}\left(a^{2} x_{01}-x_{02}\left|z_{01}\right|^{2}\right)}{\left|z_{02} \bar{z}_{01}-a^{2}\right|^{2}} .
\end{aligned}
$$

Consider the function

$$
\begin{aligned}
H= & u_{0} \kappa_{1} x_{01}\left(1-\frac{a^{2}}{x_{01}^{2}+y_{01}^{2}}\right)+u_{0} \kappa_{2} x_{02}\left(1-\frac{a^{2}}{x_{02}^{2}+y_{02}^{2}}\right) \\
& +\frac{\kappa_{1} \kappa_{2}}{2} \log \left(\left(x_{01}-x_{02}\right)^{2}+\left(y_{01}-y_{02}\right)^{2}\right)-\frac{\kappa_{1}^{2}}{2} \log \left(x_{01}^{2}\right. \\
& \left.+y_{01}^{2}-a^{2}\right)-\frac{\kappa_{2}^{2}}{2} \log \left(x_{02}^{2}+y_{02}^{2}-a^{2}\right)+\frac{\kappa_{1} \kappa_{0}}{2} \log \left(x_{01}^{2}\right. \\
& \left.+y_{01}^{2}\right)+\frac{\kappa_{2} \kappa_{0}}{2} \log \left(x_{02}^{2}+y_{02}^{2}\right)-\frac{\kappa_{1} \kappa_{2}}{2} \log \left(\left(y_{01} x_{02}\right.\right. \\
& \left.\left.-x_{01} y_{02}\right)^{2}+\left(y_{01} y_{02}+x_{01} x_{02}-a^{2}\right)^{2}\right) .
\end{aligned}
$$

Then

$$
\frac{d x_{0 i}}{d t}=\frac{1}{\kappa_{i}} \frac{\partial H}{\partial y_{0 i}}, \quad \frac{d y_{0 i}}{d t}=-\frac{1}{\kappa_{i}} \frac{\partial H}{\partial x_{0 i}}, \quad i=1,2 .
$$

Hence the system is Hamiltonian. Consider the angular momentum

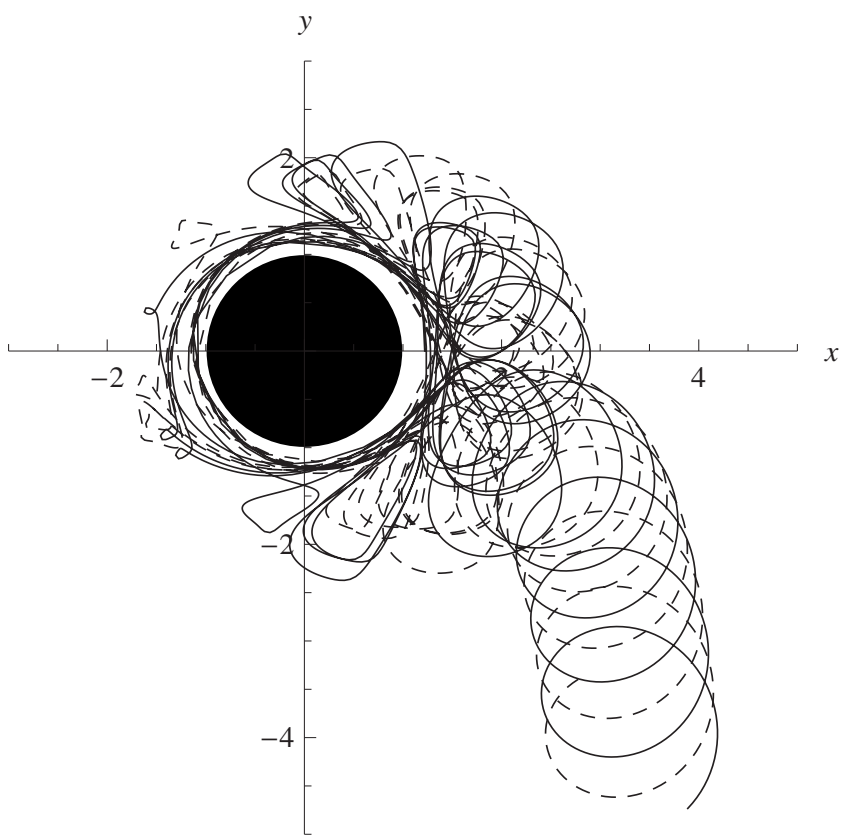

FIG. 7. Trajectories of two vortices in uniform flow $\left(u_{0}=0.1\right)$ with $\kappa_{0}=\kappa_{1}$ $+\kappa_{2}$ and initial points $z_{01}=1.2206$ and $z_{02}=2.1237$. Continuous and dashed lines denote the trajectories of vortices. The initial points correspond to point $\mathrm{C}$ in Fig. 5.

$$
I=\sum_{k=1}^{2} \kappa_{k} z_{0 k} \bar{z}_{0 k} .
$$

Since

$$
\begin{aligned}
\frac{d I}{d t}= & \frac{d}{d t}\left(\sum_{k=1}^{2} \kappa_{k} z_{0 k} \bar{z}_{0 k}\right) \\
= & \kappa_{1}\left(2 x_{01} \frac{d x_{01}}{d t}+2 y_{01} \frac{d y_{01}}{d t}\right) \\
& +\kappa_{2}\left(2 x_{02} \frac{d x_{02}}{d t}+2 y_{02} \frac{d y_{02}}{d t}\right) \neq 0,
\end{aligned}
$$

angular momentum is not independent of time and hence the system is probably not integrable.

Consider the case $\kappa_{1}=\kappa_{2}=1, \kappa_{0}=2, a=1$, and $u_{0}=0.1$, then the motion of vortices starting at $z_{01}=1.2206$ and $z_{02}=2.1237$ (corresponding to point $\mathrm{C}$ of Fig. 5) is shown in Fig. 7. We can see that initially vortices rotate around the cylinder in a chaotic fashion and then escape to infinity. It can be shown that the trajectories depend on the initial conditions drastically, for example, in Fig. 8 initial conditions are $z_{01}=1.322876$ and $z_{02}=2.061553$ (corresponding to point B of Fig. 5) and vortices rotate around the cylinder regularly as if there is no uniform flow (compare it with Fig. $3)$. It is no coincidence that in the latter case initial points correspond to a region that is inside the gray shaded zone shown in Fig. 5 and that in the former case we are just outside that zone.

A capture zone where vortices rotate around the cylinder is shown in Fig. 5. Outside this zone, vortices escape to infinity unless they start off with initial points that correspond to points close to the cylinder in Fig. 5, in which case 


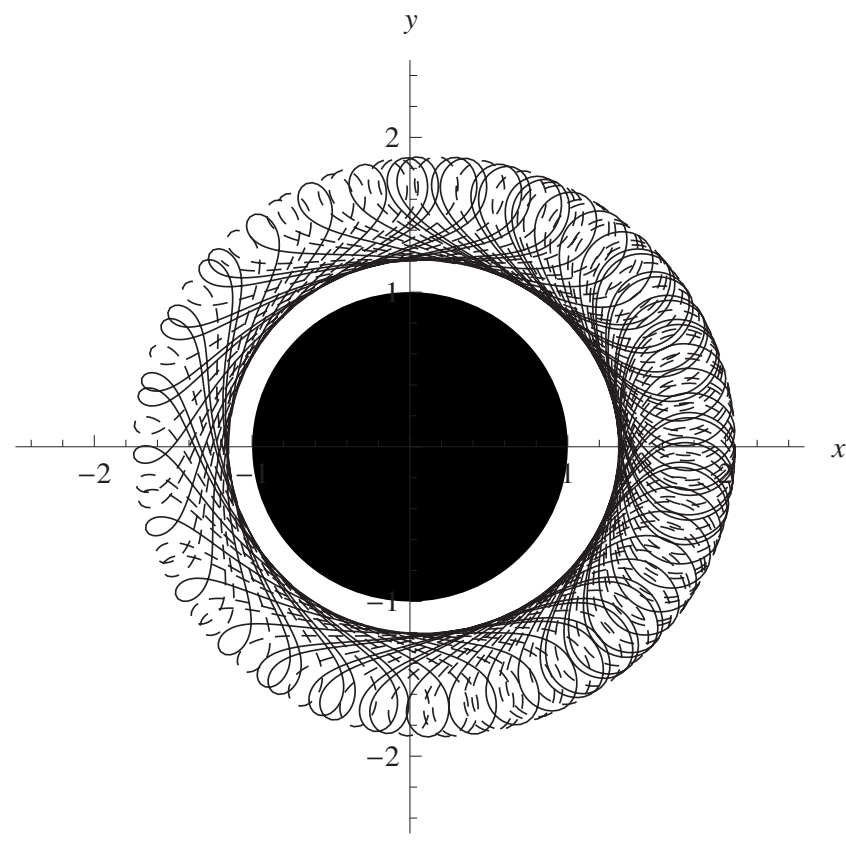

FIG. 8. Trajectories of two vortices in uniform flow $\left(u_{0}=0.1\right)$ with $\kappa_{0}=\kappa_{1}$ $+\kappa_{2}$ and initial points $z_{01}=1.322876$ and $z_{02}=2.061$ 553. Continuous and dashed lines denote the trajectories of vortices. The initial points correspond to point B in Fig. 5.

they rotate around the cylinder just like the capture zone. In fact, the choice of initial conditions are not arbitrary: for Fig. 8 initial conditions are chosen such that we are inside the capture zone in Fig. 5 (point B) and for Fig. 7 initial conditions are chosen so that we are just outside the capture zone in Fig. 5 (point C). So, we can conclude that if we are close enough to the elliptic point of the integrable case of two vortices without uniform flow, the trajectories in both cases would behave similarly (Figs. 3 and 8), but when we are far from the elliptic points (outside the capture zone), trajectories would behave differently (Figs. 3 and 7). Point E of Fig. 5 corresponds to the physical situation that vortices are placed such that the lines passing through the vortices and the origin are perpendicular. In this case vortices escape to infinity. Point A of Fig. 5 is very close to the elliptic point and the initial positions of vortices are very close to each other and the vortices rotate around the cylinder.

A Poincare section of the case with uniform flow would be helpful to analyze the system. Around the elliptical point

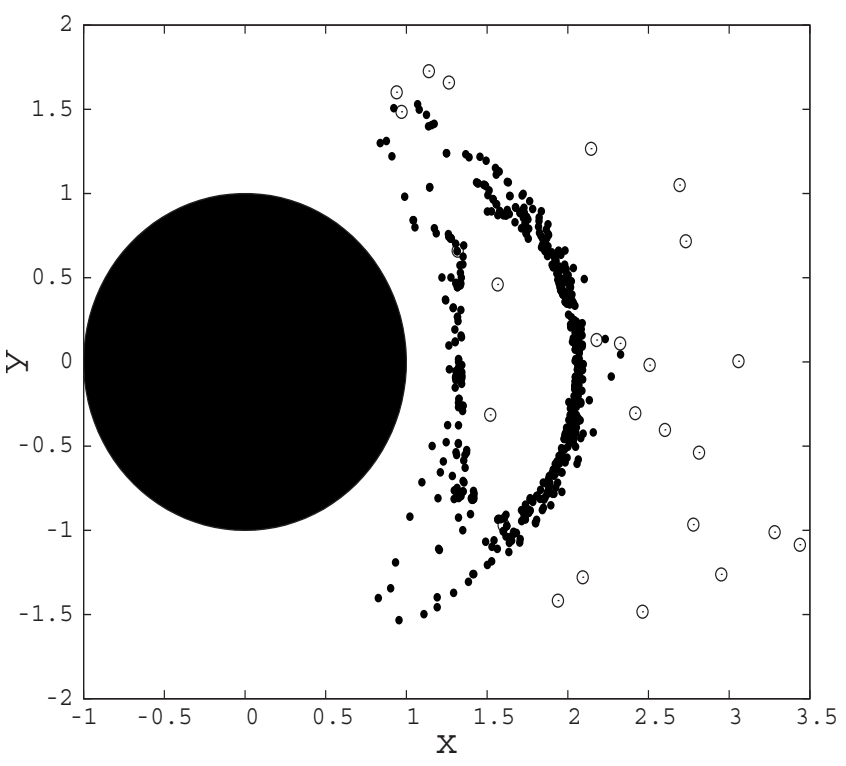

FIG. 9. Poincare section for the parameters which generates the system of Fig. 7. Solid dots are used for the five different initial conditions for which the vortices lie on invariant tori of the elliptical point. Unfilled circles are used for the sixth initial condition that vortices eventually escape to infinity after several rotations around the cylinder.

we choose six different and arbitrary initial conditions and plot the position of the second vortex when the first vortex crosses the positive part of the $x$ axis (see Fig. 9). For five initial conditions, the strobed trajectory points lie on invariant tori of the elliptical point (solid dots), while for the sixth initial condition, the points are scattered and eventually vortices escape to infinity (Fig. 7).

In all numerical simulations, the Runge-Kutta method with adaptive step size control is used to integrate the motion equations. Its results compare very well with the results of some standard packages. We notice that in almost all simulations involving chaotic behavior, it was necessary to vary the time step.

\section{OSCILLATING CYLINDER IN A UNIFORM FLOW WITH VORTICES}

The oscillating cylinder problem with only one vortex has been solved by Kadtke and Novikov. ${ }^{18}$ Here we shall consider the case of two vortices. The Hamiltonian is slightly different from the previous case,

$$
\begin{aligned}
H= & u_{0}(1+\epsilon \sin w t)\left(\kappa_{1} x_{01}\left(1-\frac{a^{2}}{x_{01}^{2}+y_{01}^{2}}\right)+\kappa_{2} x_{02}\left(1-\frac{a^{2}}{x_{02}^{2}+y_{02}^{2}}\right)\right)+\frac{\kappa_{1} \kappa_{2}}{2} \log \left(\left(x_{01}-x_{02}\right)^{2}+\left(y_{01}-y_{02}\right)^{2}\right) \\
& -\frac{\kappa_{1}^{2}}{2} \log \left(x_{01}^{2}+y_{01}^{2}-a^{2}\right)-\frac{\kappa_{2}^{2}}{2} \log \left(x_{02}^{2}+y_{02}^{2}-a^{2}\right)+\frac{\kappa_{1} \kappa_{0}}{2} \log \left(x_{01}^{2}+y_{01}^{2}\right)+\frac{\kappa_{2} \kappa_{0}}{2} \log \left(x_{02}^{2}+y_{02}^{2}\right) \\
& -\frac{\kappa_{1} \kappa_{2}}{2} \log \left(\left(y_{01} x_{02}-x_{01} y_{02}\right)^{2}+\left(y_{01} y_{02}+x_{01} x_{02}-a^{2}\right)^{2}\right),
\end{aligned}
$$




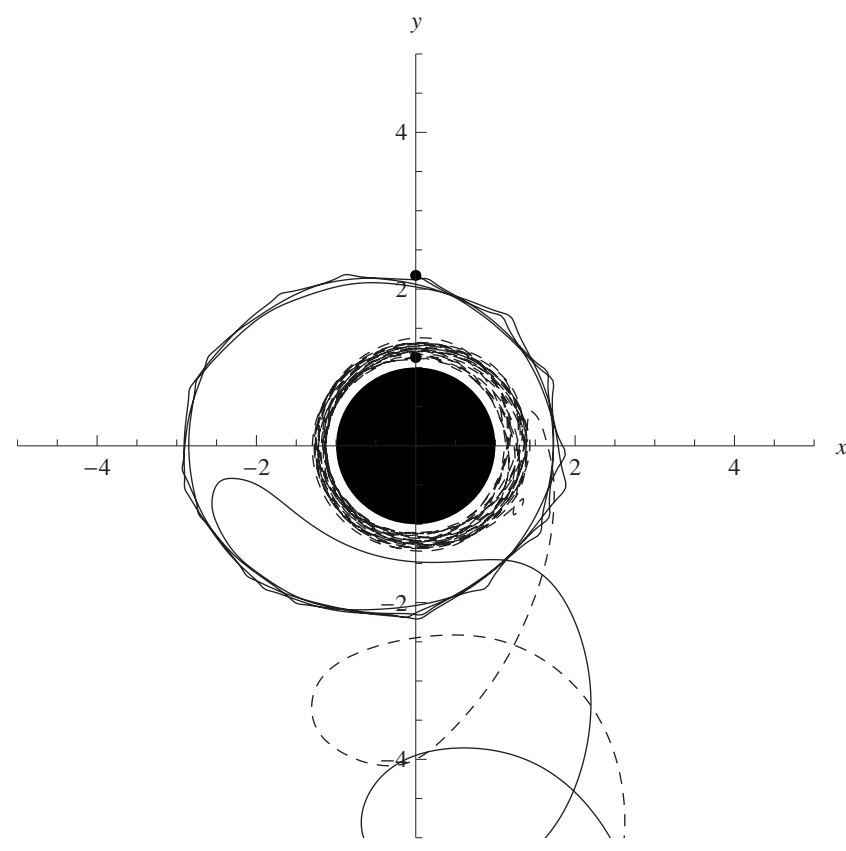

FIG. 10. Trajectories of two vortices. The parameters are $\kappa_{1}=1, \kappa_{2}=1$, $\kappa_{0}=2, \epsilon=0.1, \omega=1, u_{0}=0.1, z_{01}=(0,1.126865), z_{02}=(0,2.174897)$. Continuous and dashed lines denote the trajectories of vortices. Initial points correspond to a point just to the right of point D of Fig. 5. Small solid dots indicate the initial positions of vortices.

where $w$ is the frequency and $\epsilon$ is the amplitude of the oscillation. The motion equations can be written down using

$$
\frac{d x_{0 i}}{d t}=\frac{1}{\kappa_{i}} \frac{\partial H}{\partial y_{0 i}}, \quad \frac{d y_{0 i}}{d t}=-\frac{1}{\kappa_{i}} \frac{\partial H}{\partial x_{0 i}}, \quad i=1,2 .
$$

In Figs. 10 and 11, trajectories of the vortices are shown for different initial conditions. For all cases, vortices' initial positions are on $y$ axis: In Fig. 10 vortices are on points $z_{01}=(0,1.126865), z_{02}=(0,2.174897) \quad$ (corresponding just to the right of point $\mathrm{D}$ of Fig. 5) initially and both vortices escape to infinity eventually after both of them rotate around the cylinder many times; for the second case [Figs. 11(a) and 11(b)], vortices start at points $z_{01}=(0,1.126200), z_{02}$ $=(0,2.175241)$ and $z_{01}=(0,1.220600), z_{02}=(0,2.175241)$, respectively, and there is no escape to infinity. Initial points for Fig. 11(a) correspond to point D of Fig. 5 which is close to the cylinder, so vortices rotate around the cylinder. For Fig. 11(b), initial points correspond to point $\mathrm{C}$ of Fig. 5 and vortices rotate around the cylinder. So we conclude that if we are close enough to the cylinder [Fig. 11(a)] or to the capture zone [Fig. 11(b)], vortices rotate, however, if we are just in between these regions escape occurs (Fig. 10). So the capture zone is roughly valid (slightly enlarged) for the case of vibrating cylinder.

Chaotic interaction of a cylinder with vortices could result in large forces compared with the integrable case (Figs. 12 and 13). Magnitude of the force on the cylinder in the integrable case of two vortices is shown in Fig. 12. For chaotic cases when there is a uniform flow together with perturbation on the cylinder and two vortices (Fig. 13), the force
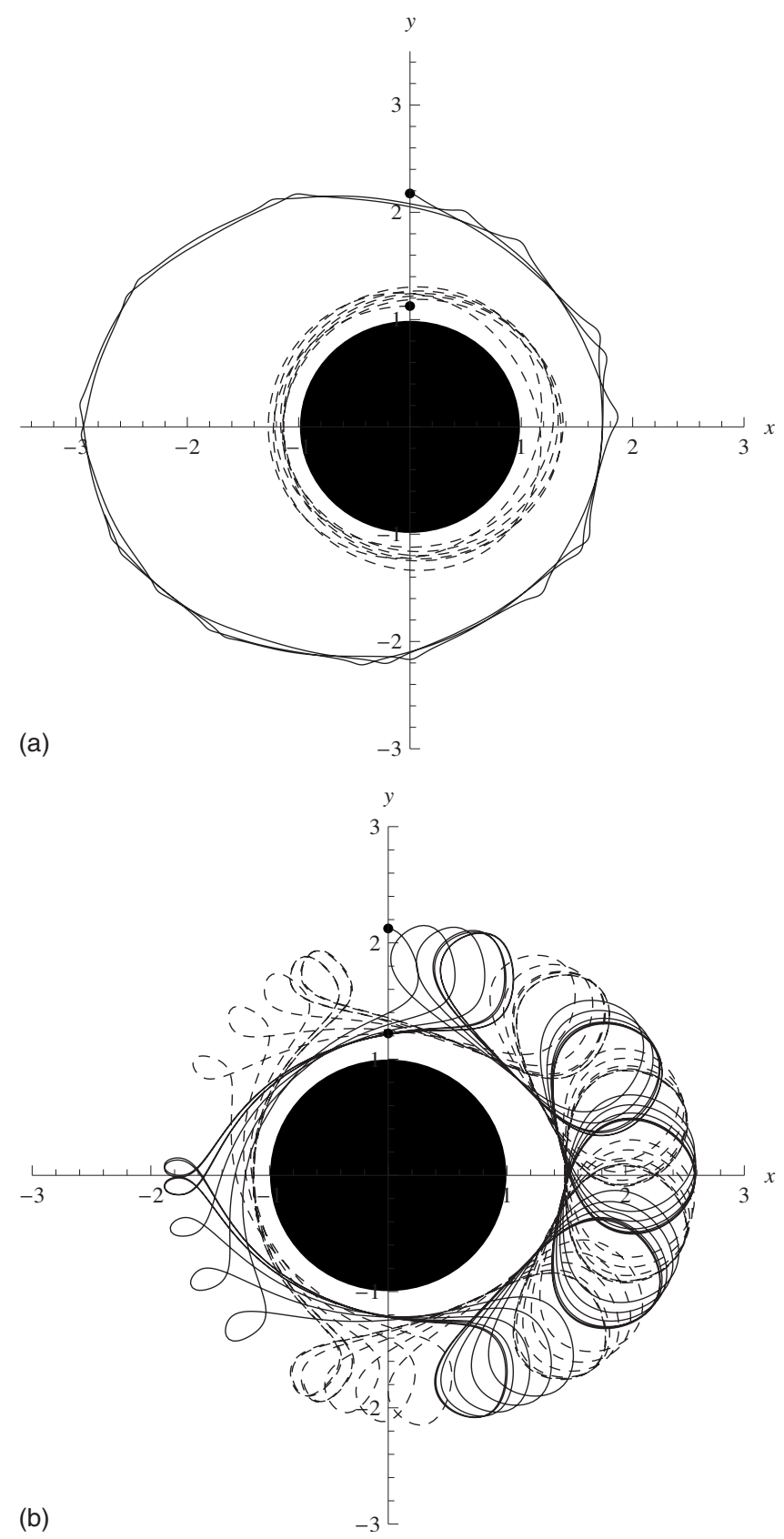

FIG. 11. (a) Trajectories of two vortices. The parameters are $\kappa_{1}=1, \kappa_{2}=1$, $\kappa_{0}=2, \epsilon=0.1, \omega=1, u_{0}=0.1, z_{01}=(0,1.126200), z_{02}=(0,2.175241)$. Continuous and dashed lines denote the trajectories of vortices. Initial points correspond to point D of Fig. 5. Small solid dots indicate the initial positions of vortices. (b) Trajectories of two vortices. The parameters are $\kappa_{1}=1, \kappa_{2}$ $=1, \kappa_{0}=2, \epsilon=0.1, \omega=1, u_{0}=0.1, z_{01}=(0,1.220600), z_{02}=(0,2.175241)$. Continuous and dashed lines denote the trajectories of vortices. Initial points correspond to point $\mathrm{C}$ of Fig. 5. Small solid dots indicate the initial positions of vortices.

on the cylinder may become twice as large as the force in the integrable case depending on the initial conditions. Similar results on pressure distribution have been obtained by Kadtke and Novikov ${ }^{18}$ for the one vortex case. This result has important consequences in some engineering applications such as the interaction of tethers of tension leg platforms with vortices and uniform flow. 


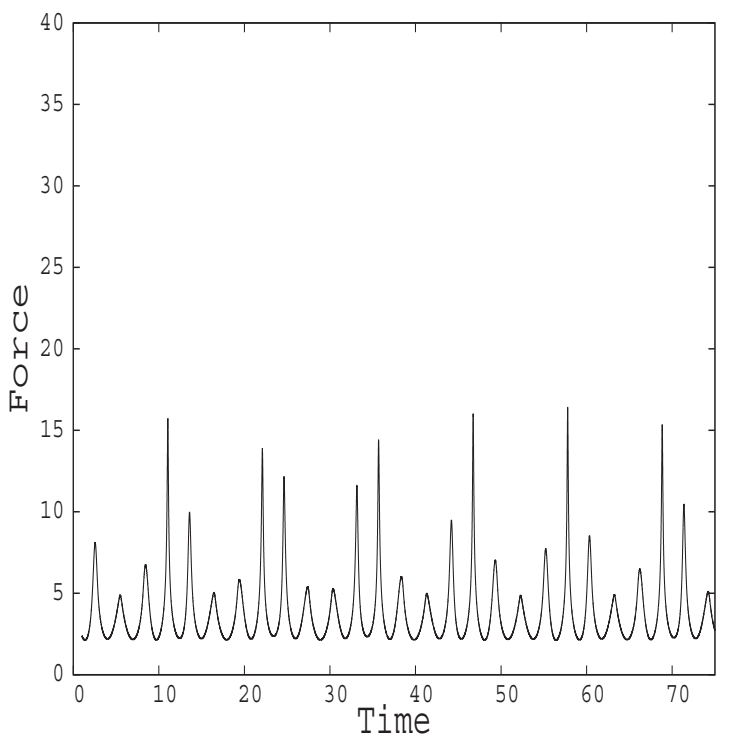

FIG. 12. Magnitude of force on the cylinder. The parameters are $\kappa_{1}=1$, $\kappa_{2}=1, \kappa_{0}=2, u_{0}=0, z_{01}=1.2206, z_{02}=2.1237$.

\section{CONCLUSIONS}

Two problems are studied: the problem of fluid advection excited by two vortices and a uniform flow in the presence of a cylinder and the problem of motion of the vortices around the cylinder where a perturbation of the cylinder is also considered. In the fluid advection problem forces are calculated. The arbitrary independent circulation around the cylinder, $\kappa_{0}$, is an important parameter in force calculations; for the case of vortices with opposite signs with or without uniform flow $\kappa_{0}$ must be zero for force to be zero.

The Hamiltonian for the motion of vortices without uniform flow is constructed, reduced, and constant Hamiltonian (energy) curves are plotted and the system is shown to be integrable according to Liouville. Motions of vortices with

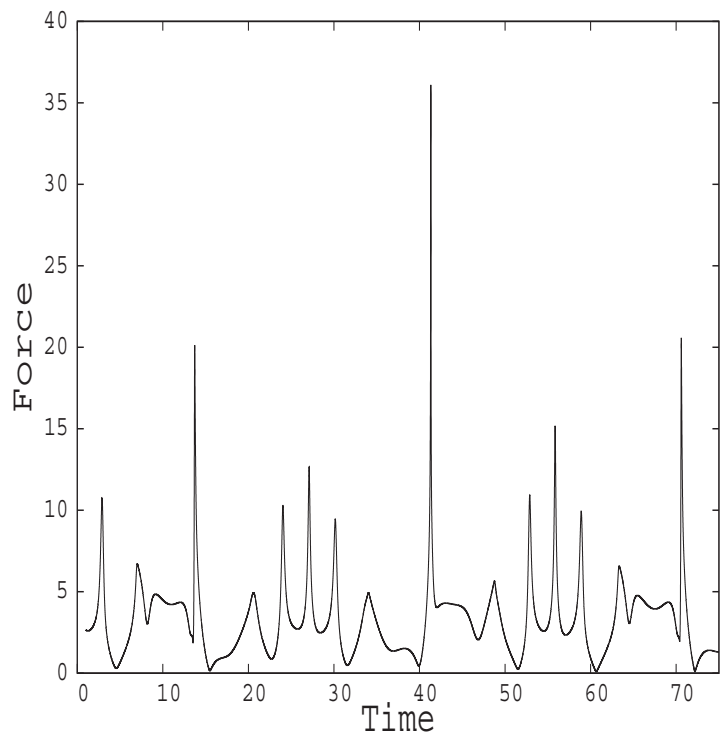

FIG. 13. Magnitude of force on the cylinder. The parameters are $\kappa_{1}=1$, $\kappa_{2}=1, \kappa_{0}=2, \epsilon=0.1, \omega=1, u_{0}=0.1, z_{01}=1.2206, z_{02}=2.1237$. unit strength are confined to a region between two concentric circles; small circle representing the cylinder and the larger one exist due to the conservation of angular momentum (Fig. 5). There are also two fixed elliptical points.

By adding uniform flow to the system, the symmetry is destroyed and the system is no longer integrable (Fig. 7). However, by choosing the initial points close to the elliptic points of the integrable case in Fig. 5, we are able to obtain trajectories confined to a thick orbit around the cylinder (Fig. 8). A Poincare section analysis was useful to demonstrate that away from the elliptic points motions are chaotic (Fig. 9).

Finally, by allowing the cylinder to vibrate we model the natural vibration of the cylinder in the flow field, which has applications in ocean engineering involving tethers or pipelines in a flow field. Interesting cases of chaotic capture and escape are shown in Figs. 10 and 11. We also observe that chaotic interaction may cause large forces on the cylinder depending on the initial positions of vortices (Figs. 12 and 13).

There are similarities and differences between the one vortex case of Kadtke and Novikov ${ }^{18}$ and the present investigation of two vortices. In both cases, chaotic capture and escape of the vortex (vortices) occur. Kadtke and Novikov gave a capture zone in Fig. 12 of their paper, which is closely related to the separatrix. In the present case of two vortices, a capture zone plot similar to the one given by Kadtke and Novikov is given in Fig. 5. When $u_{0}$ is small, say, less than 0.1 , and there is no vibration, vortices with initial points corresponding to a region inside the capture zone will not escape to infinity (see Fig. 8). So, for initial points corresponding to points that are inside the capture zone or that are close to the cylinder in Fig. 5, vortices rotate around the cylinder. Outside these two regions, vortices escape to infinity.

When there is vibration of small magnitude, $\epsilon=0.1$, and of frequency $\omega=1$, the capture zone plot is still roughly valid (see Figs. 10 and 11). For Figs. 11(a) and 11(b), the initial points correspond to points $\mathrm{D}$ and $\mathrm{C}$ of Fig. 5 and there is no escape. However, for Fig. 10, the initial points are chosen to correspond just to the right of point $\mathrm{D}$ (between $\mathrm{C}$ and $\mathrm{D}$ ) and vortices escape. We can conclude that the effect of perturbation is to enlarge the capture zone slightly.

\section{ACKNOWLEDGMENTS}

The authors are grateful to the referees, who suggested several important improvements to the presentation of the results.

${ }^{1}$ L. M. Milne-Thomson, Theoretical Hydrodynamics (Macmillan, London, 1968).

${ }^{2}$ A. K. Banik and T. K. Datta, "Stability analysis of TLP tethers under vortex induced oscillations," J. Offshore Mech. Arct. Eng. 131, 011601 (2009).

${ }^{3}$ C. H. K. Williamson and R. Govardhan, "Vortex induced vibrations," Annu. Rev. Fluid Mech. 36, 413 (2004).

${ }^{4}$ Y. Dong, G. Xie, and J. Y. K. Lou, "Stability of vortex induced oscillations of tension leg platforms," Ocean Eng. 19, 555 (1992).

${ }^{5}$ C. M. Linton and D. V. Evans, "The interaction of waves with arrays of vertical circular cylinder,” J. Fluid Mech. 215, 549 (1990).

${ }^{6} \mathrm{O}$. Yilmaz, "Hydrodynamic interactions of waves with a group of trun- 
cated vertical cylinders," J. Waterway, Port, Coastal, Ocean Eng. 124, 272 (1998).

${ }^{7}$ O. Pashaev and O. Yilmaz, "Power series solution for the two dimensional inviscid flow with a vortex and multiple cylinders," J. Eng. Math. 65, 157 (2009).

${ }^{8}$ O. Pashaev and O. Yilmaz, "Vortex images and q-elementary functions," J. Phys. A 41, 135207 (2008).

${ }^{9}$ E. R. Johnson and N. R. McDonald, "The motion of a vortex near two circular cylinders," Proc. R. Soc. London, Ser. A 460, 939 (2004).

${ }^{10}$ D. A. Burton, J. Gratus, and R. W. Tucker, "Hydrodynamic forces on two moving discs," Theor Appl. Mech. 31, 153 (2004).

${ }^{11} \mathrm{D}$. Crowdy and J. Marshall, "The motion of a point vortex around multiple circular cylinders," Phys. Fluids 17, 056602 (2005).

${ }^{12} \mathrm{H}$. Aref, "Integrable, chaotic, and turbulent vortex motion in two dimensional flows," Annu. Rev. Fluid Mech. 15, 345 (1983).
${ }^{13}$ H. Aref and N. Pomphrey, "Integrable and chaotic motions of four vortices I. The case of identical vortices," Proc. R. Soc. London, Ser. A 380, 359 (1982).

${ }^{14} \mathrm{~B}$. Eckhardt and H. Aref, "Integrable and chaotic motions of four vortices II. Collision dynamics of vortex pairs," Philos. Trans. R. Soc. London, Ser. A 326, 655 (1988).

${ }^{15}$ B. Eckhardt, "Integrable four vortex motion," Phys. Fluids 31, 2796 (1988).

${ }^{16} \mathrm{E}$. R. Johnson and N. R. McDonald, "The point island approximation in vortex dynamics," Geophys. Astrophys. Fluid Dyn. 99, 49 (2005).

${ }^{17}$ L. Zannetti and P. Franzese, "Advection by a point vortex in closed domains," Eur. J. Mech. B/Fluids 12, 43 (1993).

${ }^{18}$ J. B. Kadtke and E. A. Novikov, "Chaotic capture of vortices by a moving body I. Single point vortex case," Chaos 3, 543 (1993). 